\title{
Influence of Chaotic Dynamics on the Performance of Evolutionary Algorithms - An initial study
}

\author{
${ }^{(1)}$ Roman Senkerik, ${ }^{(2)}$ Donald Davendra, ${ }^{(1)}$ Ivan Zelinka, ${ }^{(1)}$ Zuzana Oplatkova, \\ ${ }^{(1)}$ Tomas Bata University in Zlin , Faculty of Applied Informatics, Nam T.G. Masaryka 5555, 76001 Zlin, \\ Czech Republic \\ ${ }^{(2)}$ Technical University of Ostrava, Faculty of Electrical Engineering and Computer Science, 17. listopadu 15, \\ 70833 Ostrava-Poruba, Czech Republic
}

\begin{abstract}
This paper outlines the initial investigations on the influence of chaotic dynamics to the performance of evolutionary algorithms. The focus of this paper is the embedding of chaotic system in the form of chaos number generator for Differential Evolution. The chaotic systems of interest are the discrete dissipative systems. The twodimensional Dissipative Standard map was selected as a possible chaos number generator for Differential Evolution. Repeated simulations were performed on a selected benchmark function. Finally, the obtained results are compared with canonical Differential Evolution.
\end{abstract}

Keywords: Chaos, optimization, evolutionary algorithms.

PACS: $02.30 . \mathrm{Oz}$

\section{INTRODUCTION}

During the past five years, usage of new intelligent systems in engineering, technology, modeling, computing and simulations has attracted the attention of researchers worldwide. The most current methods are mostly based on soft-computing, which is a discipline tightly bound to computers, representing a set of methods of special algorithms, belonging to the artificial intelligence paradigm. The most popular methods are neural networks, evolutionary algorithms or fuzzy logic.

Presently, evolutionary algorithms are known as a powerful set of tools for almost any difficult and complex optimization problem. Ant Colony (ACO), Genetic Algorithms (GA), Differential Evolution (DE), Particle Swarm Optimization (PSO) and Self Organizing Migration Algorithm (SOMA) are some of the most potent heuristics available.

Recent studies have shown that Differential Evolution (DE) [1] has been used for a number of optimization tasks, [2], [3] has explored DE for combinatorial problems, [4] has hybridized DE whereas [5] - [7] has developed selfadaptive DE variants.

This paper is aimed at investigating the chaos driven DE. Although a several DE variants with connection of chaotic dynamics and evolutionary or mutation/crossover process have been recently developed, the focus of this paper is the embedding of chaotic systems in the form of chaos number generator for $\mathrm{DE}$ and its comparison with the canonical DE. This research is an extension and continuation of the previous initial application based experiment with chaos driven DE [8]. The chaotic systems of interest are discrete dissipative systems.

Firstly, the used chaotic system is described. The next sections are focused on the description of benchmark test function and introduction of Differential Evolution. Results and conclusion follow afterwards.

\section{SELECTED CHAOTIC MAP}

This section contains the description of chaotic map used as the random generator for DE. Iterations of the chaotic map were used for the generation of real numbers in the process of crossover based on the user defined $C R$ value and for the generation of the integer values used for selection of solutions (individuals).

Numerical Analysis and Applied Mathematics ICNAAM 2012

AIP Conf. Proc. 1479, 627-630 (2012); doi: 10.1063/1.4756212

(C) 2012 American Institute of Physics 978-0-7354-1091-6/\$30.00 
The Dissipative Standard map is a two-dimensional chaotic map. The parameters used in this work are $b=0.1$ and $k=8.8$ as suggested in [10]. The Dissipative standard map is given in Fig. 1. The map equations are given in Eq. 1 and 2.

$$
\begin{gathered}
X_{n+1}=X_{n}+Y_{n+1}(\bmod 2 \pi) \\
Y_{n+1}=b Y_{n}+k \sin X_{n}(\bmod 2 \pi)
\end{gathered}
$$

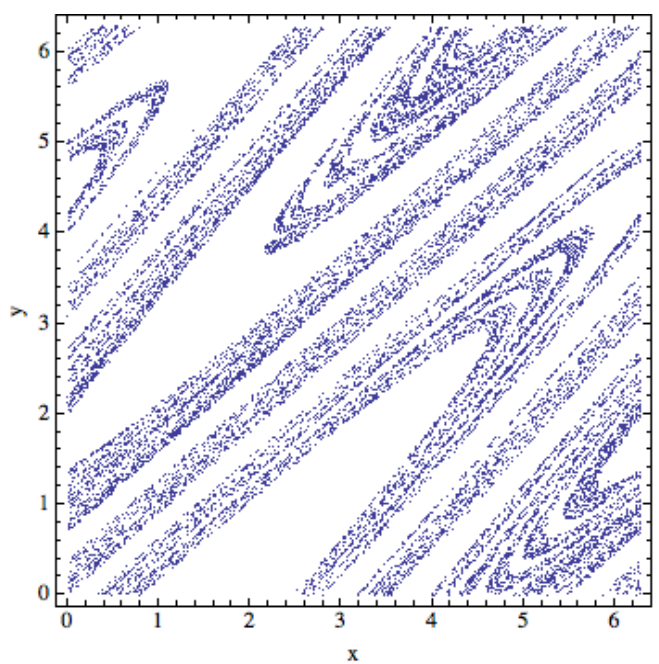

FIGURE 1. Dissipative Standard Map

\section{BENCHMARK FUNCTION}

For the purpose of evolutionary algorithms performance comparison within this initial research, the following three test functions were selected: Schwefel's function (3). The 3D diagram for $D=2$ is depicted in Fig. 2.

$$
f(x)=\sum_{i=1}^{D}-x_{i} \sin \left(\sqrt{\left|x_{i}\right|}\right)
$$

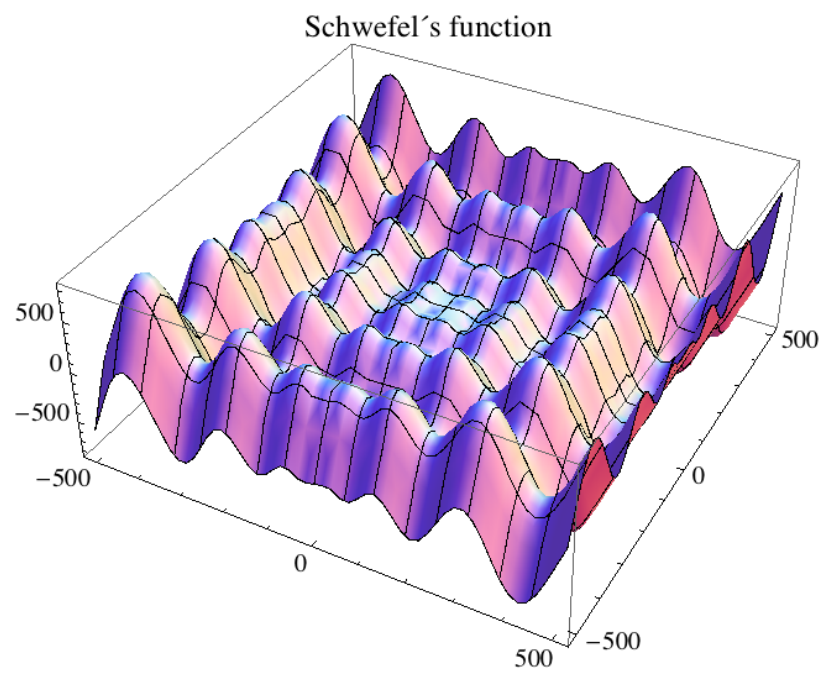

FIGURE 2. Schwefel's benchmark function. 


\section{DIFFERENTIAL EVOLUTION}

DE is a population-based optimization method that works on real-number-coded individuals [1]. DE is quite robust, fast, and effective, with global optimization ability. It does not require the objective function to be differentiable, and it works well even with noisy and time-dependent objective functions. Description of the used DEBest2Bin strategy is presented in [1], [9]. The other strategies differ in the way of calculating the perturbed vector. Please refer to [1], [9] for the detailed complete description of all other strategies.

\section{RESULTS}

The novelty of this approach represents the utilization of discrete chaotic map as a random generator for DE. In this paper, the canonical DE strategy DEBest2Bin and its ChaosDE version were used. The parameter settings for both canonical DE and ChaosDE were obtained analytically based on numerous experiments and simulations. Experiments were performed in an environment of Wolfram Mathematica, canonical DE therefore used the built-in Mathematica software random number generator. All experiments used different initialization, i.e. different initial population was generated in each run of Canonical or Chaos DE.

Within this research, two experiments were performed. The first one utilizes the maximum number of generations fixed at 200 generations. This allowed the possibility to analyse the progress of DE within a limited number of generations and cost function evaluations. In the second case, the number of generations was unlimited. The main observed parameters were the total number of cost function evaluations and the time in seconds required for finding of the global minimum of the used test functions.

The results of the first experiment are shown in Table 1, which represent the average deviations from the known global minimum for 20 repeated runs.

Table 2 contains the results for the second experiment. It show the average time in seconds and number of CFE (Cost Function Evaluations) required for the finding of exact global minimum for 20 repeated runs of the evolutionary algorithms. The bold values in both tables depict the best value.

TABLE 1. Average difference from Global minimum for the Schwefel's function: 2D - 8D

\begin{tabular}{cccc}
\hline Dimension $\boldsymbol{D}$. & Known Value & Canonical DE & ChaosDE - Dissipative \\
\hline 2 & $-837,966$ & $\mathbf{2 , 2 5 4 5 . 1 0}$ & $\mathbf{2 , 2 5 4 5 . 1 0}$ \\
4 & $-1675,932$ & 0.983358 & $\mathbf{0 . 9 7 8 3 1 2}$ \\
6 & $-2513,898$ & 370.338 & $\mathbf{3 3 8 . 0 5 6}$ \\
8 & $-3351,864$ & 928.976 & $\mathbf{8 8 0 . 2 5 5}$ \\
\hline
\end{tabular}

TABLE 2. Average CFE and evaluation time for the Schwefel's function: 10D

\begin{tabular}{lcc}
\hline & Canonical DE & ChaosDE - Dissipative \\
\hline CFE & 298200 & $\mathbf{1 7 4 4 0 0}$ \\
Time (seconds) & 157.41 & $\mathbf{1 2 9 . 7 8}$ \\
\hline
\end{tabular}

The results in Tables 1 and 2 show that using the Dissipative standard map as a random generator has actually improved the performance of DE. The performance of DE significantly improved in both experiments for the limited number of generations (2D - 8D) and for unlimited simulation (10D), which is confirmed in Fig. 3. This figure shows the evolution of the cost function value in time (iterations). Time (iterations) are converted here to a number of cost function evaluations, where in each iteration the cost function is evaluated exactly as many times as there are individuals (solutions) in the population.

\section{CONCLUSION}

In this paper, chaos driven DEBest2Bin strategy was tested and compared with canonical DEBest2Bin strategy. Based on obtained results, it may be claimed, that the developed ChaosDE driven by means of the chaotic Dissipative standard map gives better results than other compared heuristics.

Since this was an initial study, future plans include experiments with benchmark functions in higher dimensions, testing of different chaotic systems and obtaining a large number of results to perform statistical tests. 
Furthermore chaotic systems have additional parameters, which can by tuned. This issue opens up the possibility of examining the impact of these parameters to generation of random numbers, and thus influence on the results obtained using differential evolution.

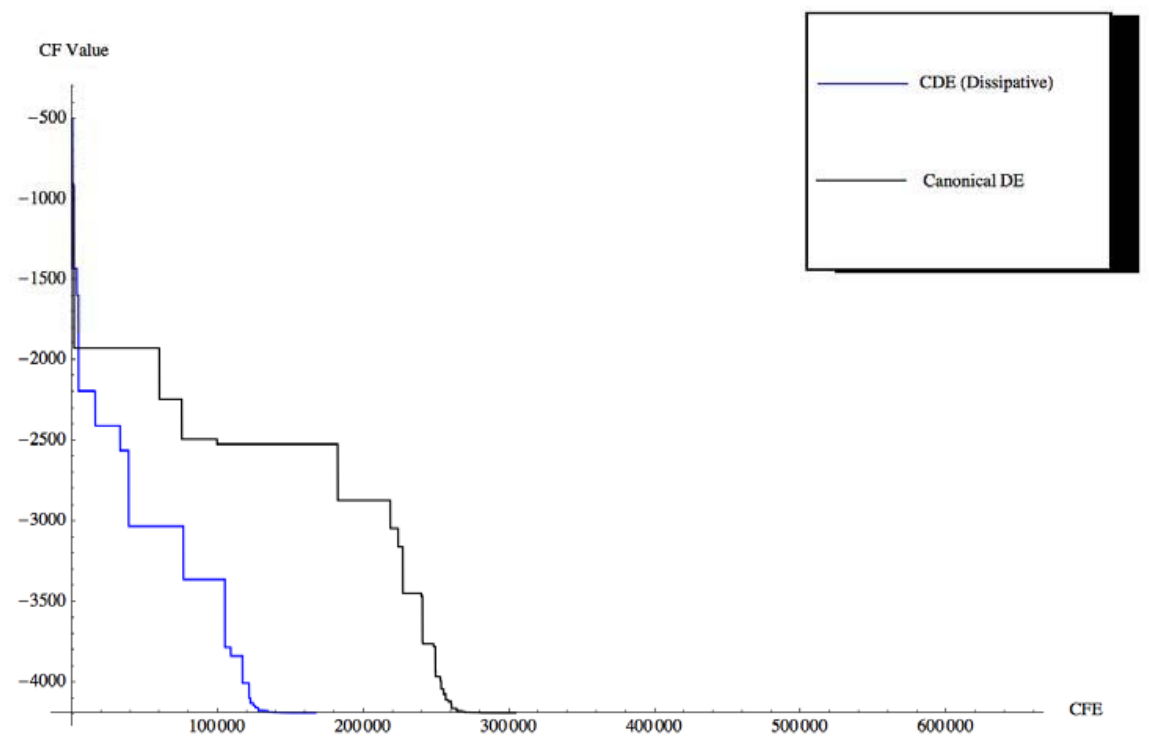

FIGURE 3. Example of the time evolution of the cost function values for Schwefel function and the second experiment - 10D.

\section{ACKNOWLEDGMENTS}

This work was supported by European Regional Development Fund under the project CEBIA-Tech No. CZ.1.05/2.1.00/03.0089.

\section{REFERENCES}

1. K. Price, "An Introduction to Differential Evolution", In: New Ideas in Optimization, (D. Corne, M. Dorigo and F. Glover, Eds.), p. 79-108, McGraw-Hill, London, UK, ISBN 007-709506-5, 1999.

2. M. F. Tasgetiren, P. N. Suganthan, Q. K. Pan, "An Ensemble of Discrete Differential Evolution Algorithms for Solving the Generalized Traveling Salesman Problem", Applied Mathematics and Computation, accepted in Oct 2009.

3. G. Onwubolu and D. Davendra (Eds), Differential Evolution: A handbook for Permutation-based Combinatorial Optimization. Springer, Germany, 2009

4. S. Das, A. Konar, U. K. Chakraborty, and A Abraham, "Differential evolution with a neighborhood based mutation operator: a comparative study", IEEE Transactions on Evolutionary Computations, 2009, vol. 13, no. 3, pp. 526-553.

5. A. K. Qin, V. L. Huang, P. N. Suganthan, Differential evolution algorithm with strategy adaptation for global numerical optimization", IEEE Transactions on Evolutionary Computations, 2009, vol. 13, no. 2, pp. 398-417.

6. J. Zhang and A. C. Sanderson, "JADE: Self-adaptive differential evolution with fast and reliable convergence performance," in Proc. IEEE Congres on Evoutionary. Computation., Singapore, Sep. 2007, pp. 2251-2258.

7. J. Zhang and A. C. Sanderson "Self-adaptive multiobjective differential evolution with direction information provided by archived inferior solutions," in Proceedings IEEE World Congress on Evolutionary. Computation, Hong Kong, China, Jun. 2008, p. $2801-2810$.

8. D. Davendra, I. Zelinka, R. Senkerik, "Chaos driven evolutionary algorithms for the task of PID control”, Computers \& Mathematics with Applications, Volume 60, Issue 4, 2010, pp 1088-1104, ISSN 0898-1221.

9. K. Price and R. Storn, Differential evolution homepage (2001) [Online]. Available: http://www.icsi.berkeley.edu/ storn/code.html

10. J. C. Sprott, "Chaos and Time-Series Analysis", Oxford University Press, 2003 
Copyright of AIP Conference Proceedings is the property of American Institute of Physics and its content may not be copied or emailed to multiple sites or posted to a listserv without the copyright holder's express written permission. However, users may print, download, or email articles for individual use. 